\title{
Formación de trampas extracelulares de neutrófilos humanos in vitro: Descripción de un método*
}

\author{
Formation of human neutrophil extracellular traps in vitro: Description of a method
}

Lizet Jazmín Pérez Zapata, ${ }^{\dagger}$ Ana María Trejos Ramírez ${ }^{\dagger}$, Andrés Augusto Arias Sierra, ${ }^{+\ddagger}$ Juan Álvaro López Quintero

\section{RESUMEN}

INTRODUCCIÓN: las trampas extracelulares de neutrófilo (NETs) son estructuras en forma de red compuestas de ADN y proteínas granulares que el neutrófilo libera al espacio extracelular en respuesta a diferentes estímulos. La NETosis es un mecanismo de muerte celular, que además cumple una función microbicida destruyendo o inhibiendo el crecimiento de microorganismos.

OBJETIVO: describir el proceso para inducir, visualizar y cuantificar la formación de NETs en neutrófilos humanos.

MÉTODOs: se aislaron neutrófilos de sangre periférica de individuos sanos y se indujo la producción de NETs utilizando diferentes concentraciones de PMA a determinados tiempos. La liberación de ADN se cuantificó por espectrofluorimetría y se evaluó la morfología de las NETs utilizando microscopía de fluorescencia. Los análisis estadísticos se realizaron con los programas SPSS 17.02 y GraphPad Prism 6.

RESULTADOS Y CONCLUSIONES: en el presente trabajo se describe un método que sirve para inducir NETs utilizando neutrófilos humanos, el cual es comparable con otros estudios descritos en la literatura. Los resultados y técnicas descritas aquí se pueden aplicar en ensayos que busquen evaluar el proceso de formación de NETs in vitro y tiene como intensión brindar herramientas metodológicas a los investigadores que deseen estudiar este proceso celular.

PALABRAS CLAVE: estandarización, neutrófilos, Nets, netosis, Phorbol 12-myristate 13-acetate, trampas extracelulares de neutrófilos.

* Este trabajo se realizó con apoyo económico de COLCIENCIAS código del proyecto 111554531412.

† Grupo de Inmunodeficiencias Primarias, Facultad de Medicina, Universidad de Antioquia, Medellín, Colombia.

$\ddagger$ Contacto: aaugusto.arias@udea.edu.co

Recepción: 28/01/2019 Aceptación: 25/03/2019

Cómo citar este artículo: Pérez-Zapata LJ, Trejos-Ramírez AM, Arias-Sierra AA, López-Quintero JA. Formación de trampas extracelulares de neutrófilos humanos in vitro: descripción de un método. Hechos Mocrobiol. 2019; 2019;10(1-2):12-22. DOI: 10.17533/udea.hm.v10n1a01 


\section{ABSTRACT}

INTRODUCTION: Extracellular Neutrophil Traps (NETs) are network structures, composed of DNA and granular proteins, which are released by neutrophils in the extracellular space in response to different stimuli. NETosis is a mechanism of cell death, which also has a microbicidal function by destroying or inhibiting the growth of microorganisms.

OBJECTIVE: To describe a procedure to induce, visualize and quantify the formation of NETs in human neutrophils.

METHODS: Peripheral blood neutrophils were isolated from healthy individuals. NETs production was induced using different concentrations of PMA at specific times. The release of DNA in the extracellular space was quantified by spectrofluorimetry, and the morphology of NETs was evaluated by fluorescence microscopy. Statistical analyses were performed using the SPSS and GraphPad Prism programs.

RESULTS AND CONCLUSIONS: This study describes a method used to induce NETs in human neutrophils, which is comparable with other studies published in scientific literature. These results can be applied in assays that seek to evaluate the formation of NETs in vitro and can hopefully be useful for researchers who wish to study this cellular process.

KEY WORDS: Standardization, Neutrophils, NETs, Netosis, Phorbol 12-myristate 13-acetate, Extracellular neutrophil traps.

\section{INTRODUCCIÓN}

Los polimorfonucleares neutrófilos (PMNN) son los leucocitos más abundantes en sangre periférica, se producen en médula ósea y una vez en circulación, su período de vida es de unas pocas horas. Son las primeras células que se reclutan y responden durante un proceso infeccioso. ${ }^{1}$ Su acción microbicida consiste en la fagocitosis y liberación de enzimas y productos proteolíticos al medio extracelular; sin embargo, recientemente se describió una nueva estrategia microbicida que a su vez constituye un nuevo modelo de muerte celular denominado NETosis. ${ }^{2}$ La NETosis es un proceso de muerte del neutrófilo diferente de apoptosis y necrosis que consiste en la liberación, al medio extracelular, de ADN, histonas y proteínas granulares en forma de redes denominadas "Trampas Extracelulares de Neutrófilos" (NETs, por sus siglas en inglés). Estas trampas tienen la capacidad de atrapar microorganismos, promover su destrucción y evitar su propagación. ${ }^{3-6}$ Las NETs juegan un doble papel a nivel inmunológico, si bien pueden servir para controlar infecciones, al mismo tiempo se han asociado con el desarrollo de autoinmunidad, vasculitis, inmunodeficiencias y procesos neoplásicos. ${ }^{7-11}$

La formación de NETs es un proceso secuencial que depende del tiempo y del tipo de estímulo utilizado. ${ }^{2,3,12}$ En primer lugar, el neutrófilo debe ser activado, algunos de los estímulos comúnmente usados son: PMA (forbol 12-miristato-13 acetato), 3,12 , interleuquinas 8 y $17,,^{13,14}$ bacterias, ${ }^{2,15}$ hongos, ${ }^{16,17}$ parásitos ${ }^{5,18}$ y virus. ${ }^{19,20}$ Una vez se induce la activación celular, el sistema NADPH oxidasa se ensambla y se inicia la síntesis de especies reactivas del oxígeno (ROS, por sus siglas en inglés), esto da lugar a la descondensación de la cromatina y la degradación de la membrana nuclear. ${ }^{21}$ Las proteínas líticas contenidas en los gránulos, tales como elastasa y mieloperoxidasa, que cumplen una función microbicida, se unen al ADN, este proceso se conoce como estadio temprano de NETosis. ${ }^{22,23}$ Finalmente se produce la degradación parcial de la membrana celular y se libera al medio extracelular el contenido de ADN y proteínas granulares formándose una estructura en forma de red que puede evidenciarse por microscopía., 2,3

En los estudios publicados sobre NETs, los procedimientos para inducir, visualizar y cuantificar las redes son muy heterogéneos y hasta la fecha no hay uniformidad entre los investigadores que acepte como "método estándar de oro" una metodología en particular. ${ }^{24-29}$ No obstante, es necesario estandarizar métodos para evaluar la formación de NETs y de esta forma garantizar la confiabilidad y reproducibilidad de los resultados. En este estudio describimos una metodología detallada para determinar la formación de NETs en humanos, y que permite una evaluación completa del proceso de NETosis que va desde la obtención y estimulación de neutrófilos humanos, hasta la visualización y cuantificación de la formación de estas estructuras. 


\section{MÉTODOS}

\section{Población de ESTUdio}

Se utilizó sangre venosa de individuos sanos, entre los 18 y 30 años. Todos los procedimientos se ajustaron a la resolución 8430 de 1993 del Ministerio de salud de Colombia. Así mismo se tuvo el aval del Comité de Bioética para la investigación en Humanos de la Sede de Investigación Universitaria de la Universidad de Antioquia.

\section{Aislamiento de Neutrófilos}

Para la extracción de PMNN se compararon dos técnicas basadas en gradiente de densidad, DextranFicoll (Sigma $10771^{\circledR}$ ) y Polymorphprep (AXISSHIELD, Norway). Para la primera, una parte de sangre anti-coagulada con citrato de sodio se mezcló con dos partes de Dextrán al $6 \%$ con $\mathrm{NaCl}$ al $0,9 \%$, la mezcla se dejó sedimentar por 45 minutos a temperatura ambiente. Pasado este tiempo, se colectó la fracción superior de células, correspondiente a leucocitos y se hizo un lavado con solución salina tamponada con fosfato (PBS) (Invitrogen). La suspensión de células se dispuso sobre el mismo volumen de Ficoll y se centrifugó a $400 \mathrm{~g}$ por 30 minutos a $4^{\circ} \mathrm{C}$, se colectó la capa de PMNN y se hizo un lavado adicional con PBS. En la segunda técnica, sangre anti-coagulada con EDTA se depositó en una relación 1:1 sobre Polymorphprep y se centrifugó a 500 g por 40 minutos a temperatura ambiente. Posteriormente, se colectó la porción de PMNN y se realizó un lavado con PBS. Finalmente, para ambas técnicas, los neutrófilos se suspendieron en RPMI 1640, se evaluó el rendimiento de cada técnica según el número de neutrófilos extraídos por mililitro de sangre y se determinó la viabilidad de estas células con azul de tripano.

\section{INDUCCIÓN DE NET}

En una cámara de cultivo Lab-Tek (Termo Fisher, ref 154534), se dispusieron en cada pozo $2.5 \times 10^{5}$ PMNN suspendidos en RPMI 1640. Las células se dejaron adhiriendo por 30 minutos a $37^{\circ} \mathrm{C}$ en $5 \%$ de $\mathrm{CO}_{2}$. Pasado este tiempo, se adicionó PMA a concentraciones de 25, 50 y $100 \mathrm{nM}$ y como control negativo se usaron células sin estímulo. Las células se incubaron por 3 horas con el fin de determinar los cambios morfológicos que se presentan durante este tiempo. De forma conse- cutiva, cada 30 minutos hasta el final de la incubación, los pozos se fijaron con paraformaldehído al $4 \%$. La tinción del ADN se hizo con Sytox Green (Molecular Probes) a una concentración final de $2.5 \mu \mathrm{M}$. Las placas se observaron con un microscopio de fluorescencia (Olympus BX60) y las imágenes se obtuvieron con una cámara Zeiss AxioCam Color tipo 412312.

\section{INMUNODETECCIÓN DE LA ELASTASA DE NEUTRÓFILO}

Para la determinación de la elastasa extracelular liberada durante la formación de las NETs, los PMNN estimulados con $50 \mathrm{nM}$ de PMA por 3 horas, se fijaron con $4 \%$ de paraformaldehído a temperatura ambiente. Posteriormente las células se bloquearon con una solución de PBS suplementada con $2 \%$ de albúmina sérica bovina (ASB), $2 \%$ de suero de cabra y $0,5 \%$ de Tween-20 durante 1 hora a temperatura ambiente. A continuación, se adicionó el anticuerpo anti-elastasa de neutrófilo (Abcam ab21595) en una proporción 1:80 y se dejó incubar una hora a temperatura ambiente, se realizó un lavado con PBS y se incubaron las células con el anticuerpo secundario conjugado con Alexa fluor 488 (Invitrogen 11008) en una proporción de 1:400; ambos anticuerpos se utilizaron disueltos en PBS suplementado con ABS al $2 \%$. Luego se realizó la tinción del ADN utilizando $5 \mu \mathrm{g} / \mathrm{mL}$ de Hoecsht 33432 (Molecular Probes H1399) durante 15 minutos. Después de tres lavados de 5 minutos con PBS a temperatura ambiente, las placas se cubrieron con una gota de Prolong Gold Antifade (Molecular Probes) y se observaron en un microscopio de fluorescencia (Olympus BX60). Las imágenes se obtuvieron con una cámara Zeiss AxioCam Color tipo 412-312 y su edición se hizo mediante el programa Photoshop CS5.1.

\section{CuANTificación de las NETs}

$2.5 \times 10^{5}$ PMNN resuspendidos en RPMI 1640 fueron dispuestos por pozo en platos negros de 96 pozos (Greiner Cell Star, Sigma MO562) y estimulados con 25, 50 y $100 \mathrm{nM}$ de PMA, y como control negativo se utilizaron células sin estímulo. Se adicionó a cada pozo $5 \mathrm{uM}$ de Sytox Green. Las células se incubaron a $37^{\circ} \mathrm{C}$ en $5 \%$ de $\mathrm{CO}_{2}$ durante 5 horas. La fluorescencia del Sytox Green se cuantificó cada 60 minutos en un espectrofluorómetro SpectraMax Gemini EM utilizando una longitud de onda de excitación de 488 $\mathrm{nm}$ y emisión de $510 \mathrm{~nm}$ a $37^{\circ} \mathrm{C}$. El $100 \%$ de fluores- 
cencia de ADN emitida por las células se determinó adicionando $0,3 \%$ de Tritón X-100 a pozos con los diferentes tratamientos. Con el fin de comprobar la presencia de ADN extracelular en el medio, se adicionaron $0.017 \mathrm{U} / \mu \mathrm{L}$ de DNasa (Invitrogen AM2239) después de 2 horas de estimulación. Después de cada lectura, las células se incubaron nuevamente a $37^{\circ} \mathrm{C}$ en $5 \%$ de $\mathrm{CO}_{2}$. Estos resultados fueron expresados como Unidades Relativas de Fluorescencia (URF). Por último, para cuantificar el porcentaje de ADN extracelular se utilizó la siguiente formula. ${ }^{30}$

$$
\begin{aligned}
& \% \text { ADN total } \\
& =\frac{\begin{array}{l}
\text { ((fluorescencia de células con PMA) - } \\
\text { (fluorescencia de células con DNasa) }
\end{array}}{\text { fluorescencia de células con Triton }} \times 100
\end{aligned}
$$

\section{AnÁlisis estadístico}

Para el análisis de los resultados se emplearon los programas GraphPad Prism 6 y SPSS Statistics 17.0.2. La diferencia de medianas se evaluó mediante $\mathrm{U}$ de Mann Whitney y $\mathrm{H}$ de Kruskal Wallis. Un valor de $P$ menor de 0,05 se consideró como criterio para determinar una diferencia estadísticamente significativa.

\section{RESULTADOS}

\section{EFICIENCIA EN LA SEPARACIÓN DE NEUTRÓFILOS}

La separación de los PMNN fue más eficiente utilizando el Polymorphprep; como se presenta en la Tabla 1. El número de neutrófilos obtenido por mililitro de sangre utilizada fue aproximadamente el doble con respecto al obtenido mediante el método Dextrán $6 \%$ y Ficoll 1077 (valor P 0,000). Por su parte, la viabilidad celular con ambos métodos fue similar, ambas con un rango entre el $94 \%$ y $100 \%$ de células vivas. El tiempo para realizar la separación celular con Polymorphprep fue de aproximadamente una hora, mientras que con el método de Dextrán Ficoll 1077 el procedimiento tomó en promedio 2 horas y 30 minutos.

\section{FORMACIÓN DE NETs INDUCIDA POR ACCIÓN DE PMA.}

La producción de NETs fue proporcional al aumento en la concentración de PMA y al tiempo de estimulación (Fig. 1). Con la concentración de $25 \mathrm{nM}$ de PMA se observa como a los 30 minutos la mayoría de las células aun conservan su morfología nuclear típica y son similares a las células sin estímulo, al cabo de 90 minutos, el núcleo de algunas células empieza a verse descondensado, lo que correspondería a lo descrito como fase temprana en la formación de NETs. Se observa, en algunos casos, ADN en el espacio extracelular formando estructuras compatibles morfológicamente con NETs; se ven como redes de ADN que se extienden desde el núcleo de la célula hacia el exterior con longitudes variables; después de 150 minutos se observa una mayor cantidad de estas estructuras en el espacio extracelular. Por su parte, con $50 \mathrm{nM}$ de PMA, el grado de estimulación es mayor, a los 30 minutos se aprecia una escasa liberación de ADN que aumenta con el tiempo de incubación, hasta llegar a los 150 minutos, cuando se observa gran cantidad de redes que cubren todo el campo visual. Finalmente, con 100 nM de PMA, la liberación de NETs fue mucho más evidente; desde los 30 minutos de incubación se aprecian redes que después de 90 minutos ocupan toda la placa con estructuras de gran tamaño y neutrófilos aglomerados. Durante todos los intervalos de tiempos evaluados el control negativo no sufrió cambios apreciables en la morfología nuclear.

Tabla 1. Características de desempeño de los métodos empleados para el aislamiento de neutrófilos (PMNN)

\begin{tabular}{|l|c|c|c|}
\hline \multicolumn{1}{|c|}{ MÉTODO } & Numero de PMNN obtenidos a partir de $1 \mathrm{~mL}$ de sangre & Valor $\mathbf{P}$ & Viabilidad IC 95\% \\
\hline Dextrán/Ficoll $1077(\mathrm{n} 9)$ & $1,1 \times 106 .(0,9 \times 106-1,4 \times 106)$ & 0,000 & $94-99 \%$ \\
\hline Polymorphprep $(\mathrm{n} 12)$ & $2,4 \times 106(2,0 \times 106-2,9 \times 106)$ & $96-100 \%$ \\
\hline
\end{tabular}




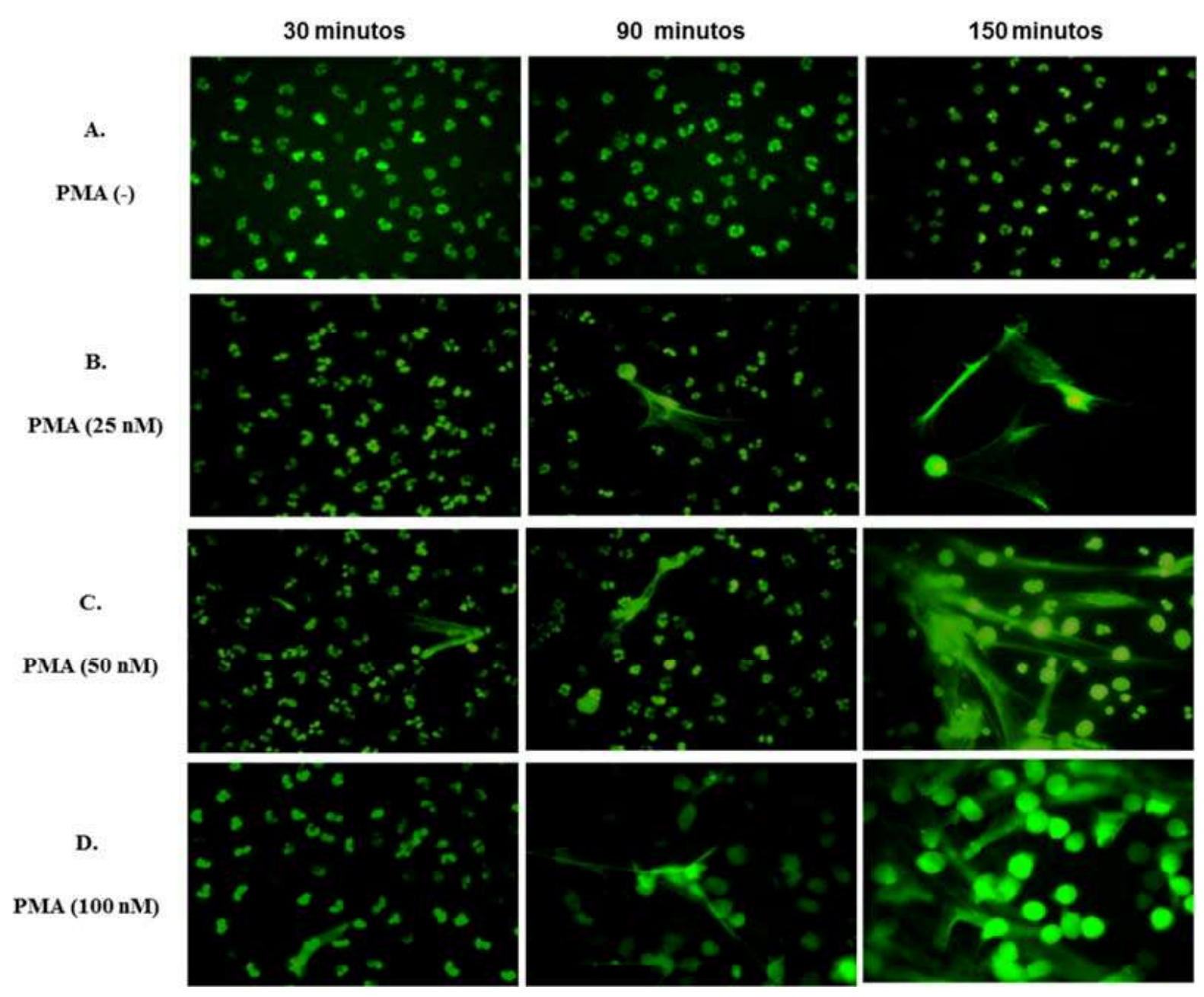

Figura 1. Formación de NETs después de la estimulación con PMA en neutrófilos humanos. Imágenes representativas de 3 repeticiones. Se observa la presencia de ADN intra y extracelular mediante tinción con Sytox Green, se evidencian los cambios morfológicos que se presentan en el neutrófilo durante el proceso de liberación de NETs a los 30, 90 y 150 minutos. A, Neutrófilos sin estímulo. B, Neutrófilos estimulados con 25 nM de PMA. C, Neutrófilos estimulados con 50 nM de PMA. D, Neutrófilos estimulados con 100 nM de PMA. Todas las imágenes fueron obtenidas mediante microscopía de fluorescencia con un aumento de 40X.

\section{Co-localización de elastasa con ADN CONFirma La PRE- SENCIA DE NETS}

Durante el proceso de formación y liberación de NETs la proteína granular elastasa y el ADN se fusionan y son liberados conjuntamente al espacio extracelular. En las células no estimuladas, la elastasa se observa en la porción citoplasmática de la célula mientras el ADN permanece todo el tiempo en la región nuclear (Fig. 2). En los neutrófilos estimulados con PMA, la elastasa se observa tanto en el espacio intra como extracelular, se puede apreciar como se localiza en la misma región que ocupa el ADN descondensado al interior de la célula, así como en el ADN liberado al medio extracelular. 
A. HOESCHST
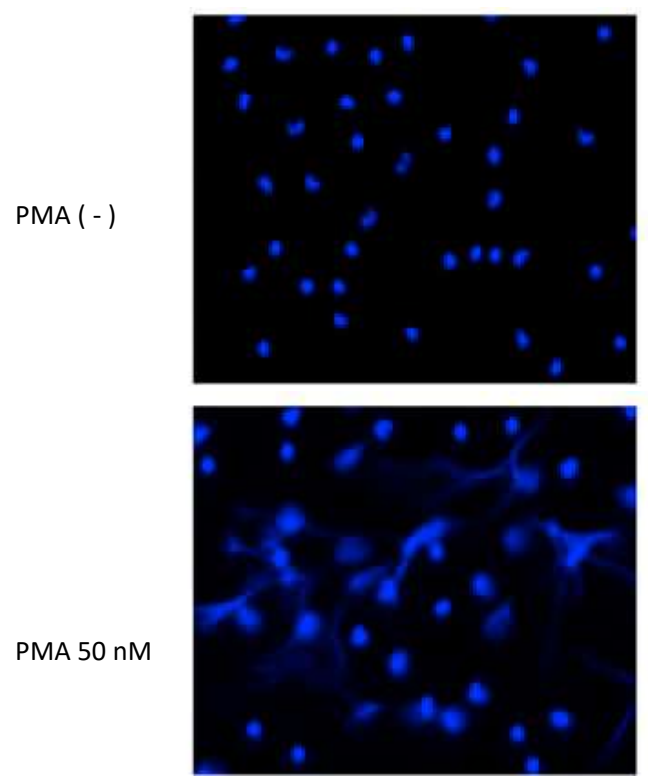

ANTI-ELASTASA ALEXA 488
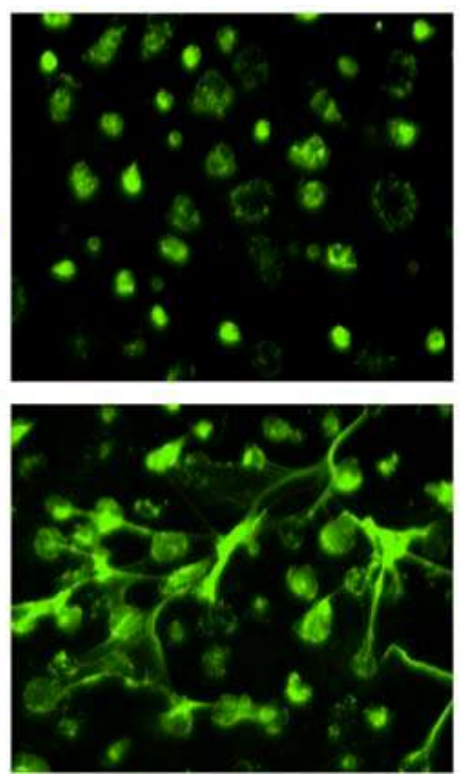
SUPERPOSICIÓN

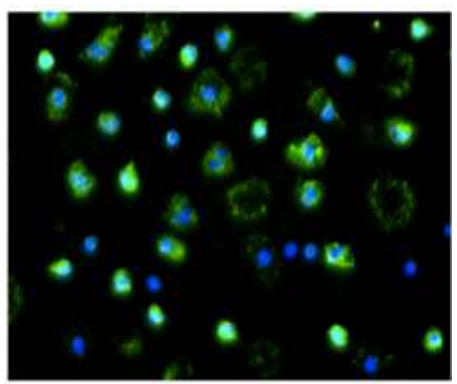

PMA 50 nM

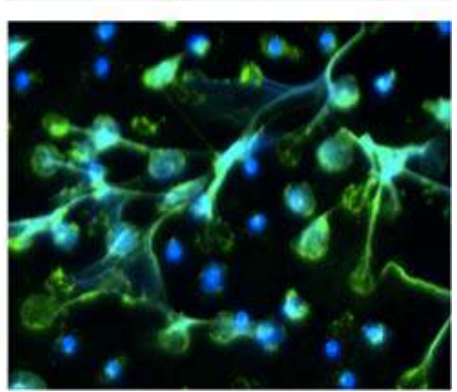

Figura 2. Identificación de NETs mediante anticuerpos anti-elastasa en neutrófilos estimulados con PMA. A, se observa la ubicación del ADN en neutrófilos teñidos con Hoeschst (Azul) que fueron tratados con $50 \mathrm{nM}$ de PMA o sin tratamiento. B corresponde al mismo campo de células, marcadas con el anticuerpo anti-elastasa acoplado al fluorocromo Alexafluor 488 (verde). C corresponde a la superposición de las imágenes observadas con Hoeschst y anti-elastasa. Todas las imágenes fueron obtenidas mediante microscopía de fluorescencia con un aumento de $63 X$.

\section{CuANTIFICACIÓN DE NETS POR ESPEC- TROFLUORIMETRÍA}

La cantidad de ADN liberado al medio extracelular fue proporcional al tiempo de estimulación, pero no a la concentración del estímulo. Como se observa en la figura 3, la concentración de ADN extracelular se detecta por espectrofluorimetría desde la primera hora de estimulación y aumenta exponencialmente hasta las 4 horas; finalmente, al término de las 5 horas se libera todo el contenido nuclear al medio, en este punto se iguala la cantidad de $\mathrm{ADN}$ al de las células tratadas con tritón. Durante todo el tiempo de evaluación, el control negativo conservó bajos niveles de fluorescencia, encontrándose diferencias significativas entre este y las células estimuladas con PMA a partir de la tercera hora $(P<0,0001)$. Sin embargo, por este medio de cuantificación no se encontraron diferencias significativas cuando se compararon entre si las concentraciones de PMA $(p>0,05)$. 

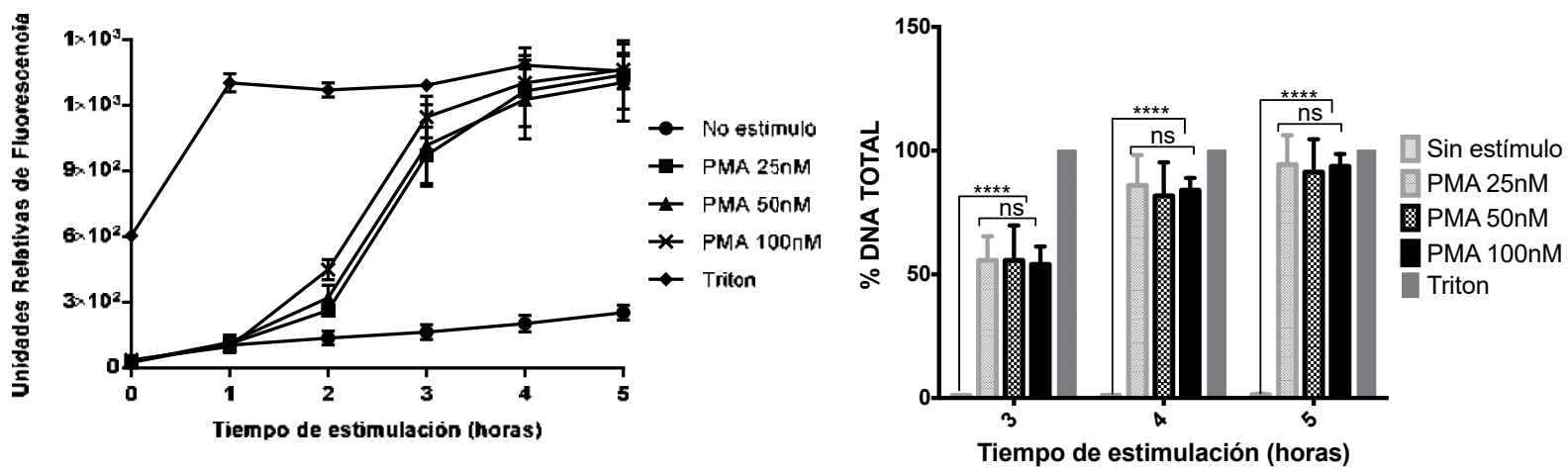

Figura 3. Cuantificación de NETs mediante espectrofluorimetría. A, Neutrófilos estimulados con 25, 50 y $100 \mathrm{nM}$ de PMA, por un tiempo máximo de 5 horas, cada hora se hizo la medición de fluorescencia de ADN unido a Sytox Green. Se utilizó $0.3 \%$ de Triton-X100 como medida del $100 \%$ de fluorescencia emitido por las células. En la figura se observan las Unidades Relativas de Fluorescencia (URF) detectadas en neutrófilos no estimulados $(\bullet)$, y estimulados con PMA $25 \mathrm{nM}(\mathbf{\square}), 50 \mathrm{nM}(\mathbf{\Delta})$ y $100 \mathrm{nM}(+)$, o tratadas con Triton-X100 ( $)$ durante el mismo tiempo. La liberación de ADN empieza a ser estadísticamente diferente del control negativo a partir de las 3 horas de estimulación y continúa hasta las 5 horas. ${ }^{* * *} P \leq 0.0001$. B, Comparación del porcentaje de ADN liberado por los neutrófilos al medio en forma de NETs después de la estimulación con $25 \mathrm{nM}, 50 \mathrm{nM}$ y $100 \mathrm{nM}$ de PMA durante 3,4 y 5 horas. El porcentaje de ADN liberado con cada concentración del estímulo es significativamente mayor al del control negativo, pero no se encontraron diferencias al comparar entre las concentraciones de PMA empleadas. ${ }^{* * * *} P \leq 0.0001{ }^{* *} P \leq 0.01$, ns: no significativo $(\mathrm{n}=3)$.

\section{La DNASA DEGRada Las NETs}

$\mathrm{Al}$ adicionar la enzima DNasa al medio, esta degrada el contenido nuclear que se encuentre fuera de la célula. Como se puede observar en la figura 4, en presencia de DNasa, en las células estimuladas con $50 \mathrm{nM}$ de PMA, se produjo una disminución signi- ficativa en la cuantificación del ADN en todos los tiempos de medición. En las células sin estímulo no se encontraron diferencias significativas después de la adición de la enzima, lo que confirma que la fluorescencia se debe al ADN liberado al exterior de la célula en forma de NETs.

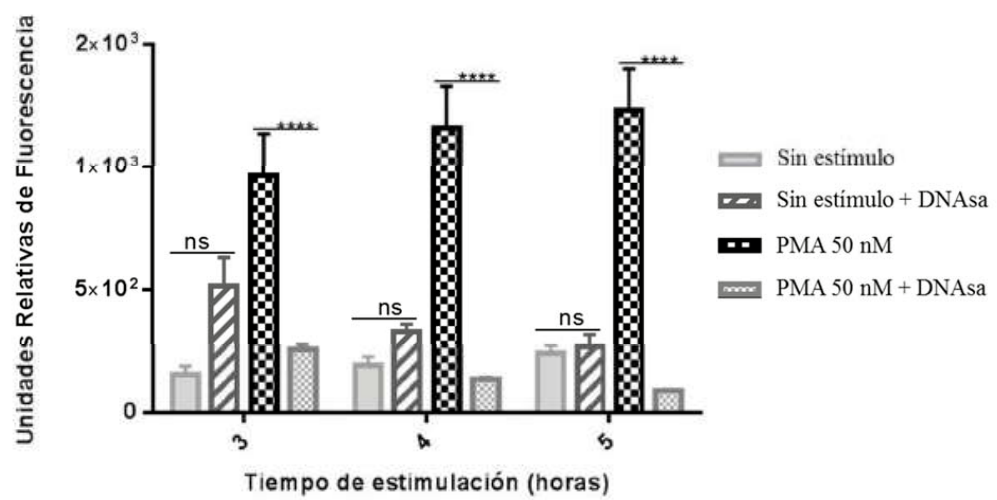

Figura 4. Degradación de NETs por acción de la DNasa. Neutrófilos estimulados con 50 nM de PMA, durante 3,4 y 5 horas. Después de dos horas de estimulación se adicionó la enzima ADNasa para degradar el ADN extracelular presente en el medio. Luego de adicionar la ADNasa, la cantidad de fluorescencia del ADN disminuyó significativamente en los neutrófilos estimulados con PMA, pero no en las células del control negativo. ${ }^{* * *} P<0.0001$. ns: No significativo $(\mathrm{n}=3)$ 


\section{DISCUSIÓN}

El presente estudio describe una metodología con la cual se induce, visualiza y cuantifica las trampas extracelulares de neutrófilos humanos, y puede usarse como una guía para la elaboración y estandarización de protocolos en la evaluación de NETs. Este proceso celular esta siendo ampliamente estudiado para dilucidar su importancia y posibles implicaciones en procesos infecciosos, enfermedades autoinmunes como el lupus eritematoso sistémico, complicaciones como la vasculitis, el cáncer e inmunodeficiencias. ${ }^{7-11} \mathrm{El}$ desarrollo de procedimientos como el aquí descrito, que permiten obtener estas redes in vitro, es el primer paso en cualquier investigación que pretenda adentrarse a entender este fenómeno celular.

El proceso completo de inducción y formación de NETs tarda entre 3 y 5 horas $^{3}$, es recomendable utilizar un método de separación de PMNN eficiente para disminuir el riesgo de activación de las células por vías diferentes a las deseadas. En nuestro caso, el método en el que se utilizó el Polymorphprep fue el que más se ajustó a estas características, la obtención de los PMNN tardó solo una hora, se obtuvo un mayor número de neutrófilos y la viabilidad de las células fue cercana al $100 \%$. Es importante mencionar que existen otros métodos de separación de neutrófilos humanos que pueden ser revisados en la literatura. ${ }^{31,32}$

ElPMA es un activador de la proteína quinasa $\mathrm{C}$, y se ha descrito como un potente inductor de la formación de NETs. ${ }^{2,3}$ Sin embargo, no está totalmente claro cuál es su papel en este proceso celular. Se sabe que activa la producción de ROS por medio del sistema NADPH oxidasa ${ }^{33,34}$ y a su vez se ha demostrado que estos compuestos oxidantes son indispensables para la NETosis. ${ }^{35,36}$ Fush y colaboradores en el 2007, demostraron que neutrófilos de pacientes con mutaciones en el sistema NADPH oxidasa eran incapaces de producir NETs ${ }^{3}$ y luego Bianchi en 2009 reportó, como después de terapia génica estos pacientes restauraban la capacidad de formar $\mathrm{NETs}^{16}$, también se ha demostrado que la inhibición de la producción de ROS previene la formación de NETs. ${ }^{3,37}$ Sin embargo, estudios recientes sugieren la existencia de mecanismos independientes de ROS para el proceso de NETosis. ${ }^{38,39}$
Las NETs se componen de fibras de cromatina de aproximadamente $15 \mathrm{~nm}$ con dominios de proteínas de $25 \mathrm{~nm}$ que alcanzan diferentes longitudes ${ }^{2}$ y en general las características morfológicas observadas en nuestro estudio son compatibles con lo publicado por otros autores. ${ }^{12,40,41}$ Nosotros evidenciamos, por medio de microscopía, que cuando se incrementó la concentración de PMA y el tiempo de estimulación, mayor fue la cantidad de redes extracelulares presentes; estos resultados son consistentes con varios autores que han reportado que la liberación de NETs por acción de PMA depende de la concentración y el tiempo de estimulación. ${ }^{2,42}$ Sugerimos que si se desea hacer una caracterización morfológica de NETs usando PMA como estímulo se usen concentraciones entre 25 y $50 \mathrm{nM}$, dado que, con concentraciones mayores, como $100 \mathrm{nM}$, las células liberan gran cantidad de NETs y tienden a formar acúmulos. Un tiempo de estimulación entre 120 y 150 minutos es suficiente para inducir la formación de estas redes extracelulares en PMNN humanos.

La observación de ADN fuera de la célula mediante microscopía no es suficiente para confirmar la presencia de NETs, si bien el ADN es la molécula más abundante de estas redes, siempre se encuentra acompañado de proteínas ${ }^{21,23,43}$ y debido a que estructuras como fibrina pueden ser confundidas con NETs, se hace imprescindible comprobar la presencia de al menos una proteína en asocio con el ADN extracelular. ${ }^{44}$ En nuestro caso comprobamos la presencia elastasa, una proteína contenida en los gránulos citoplasmáticos del neutrófilo, que además ha sido descrita como un componente fundamental en la estructura y formación de las NETs puesto que juega un papel fundamental en la citrulinación de las histonas, lo que favorece la descondensación del ADN. . $22,23^{2}$

La cuantificación de NETs por medio de espectrofluorimetría permite evaluar la cantidad de ADN extracelular liberado por los PMNN cuando se estimulan con PMA. La emisión de fluorescencia del Sytox Green, un intercalante de ADN impermeable en células vivas, incrementa cuando se une al ADN y esta característica permite cuantificar la cantidad de ADN extracelular presente en una muestra. ${ }^{45}$ Evidenciamos que la formación de NETs se presenta de manera exponencial aproximadamente hasta las 4 horas de estimulación y a partir de este punto la liberación 
de ADN tiene un comportamiento más lento, debido probablemente a que se ha liberado casi el 100\% de ADN contenido en la célula, la intensidad de fluorescencia en tiempos más prolongados de estimulación fue comparable con la de las células lisadas por tritón. Bajo el efecto del PMA, las células liberan el ADN a medida que van produciendo NETs, por su parte el tritón lisa las células desde el comienzo del estímulo y representa la fluorescencia total que se puede alcanzar en cada punto de medición.

En el primer reporte de formación de NETs en humanos se demostró, por el mismo método de espectrofluorimetría, que la producción de NETs era dependiente de la concentración de $\mathrm{PMA}^{2}$; sin embargo, en nuestro estudio no encontramos diferencias estadísticamente significativas cuando comparamos tres concentraciones de PMA, este hallazgo también se ha reportado en otros trabajos que han utilizado este mismo método de cuantificación. ${ }^{46,47}$ Probablemente, la sensibilidad del método de espectrofluorimetría no permite diferenciar claramente entre concentraciones similares a las empleadas en nuestro estudio, mientras que la microscopia si permite evidenciar cambios morfológicos diferenciales en función de la concentración de PMA. Es de anotar, que la cuantificación de NETs por microscopia está sujeta a la variación dada por la subjetividad del analista. Por lo anterior, se ha convertido en una necesidad desarrollar estrategias alternativas para cuantificar la formación de NETs de manera automática, recientemente se ha reportado el uso de citometría de flujo, ${ }^{27,29}$ y análisis visuales con procesadores de imágenes, ${ }^{24,48,49}$ entre otros. ${ }^{28,47}$

\section{CONCLUSIONES}

En conclusión, en el presente trabajo se logró describir un método que sirve para inducir la formación de NETs utilizando neutrófilos humanos, el cual es comparable con otros estudios descritos en la literatura. Los resultados obtenidos aquí se pueden aplicar en ensayos que busquen evaluar el proceso de formación de NETs in vitro en neutrófilos humanos y esperamos sea de ayuda para investigadores que deseen estudiar este proceso celular.

\section{CONFLICTO DE INTERÉS}

Los autores declaran la inexistencia de conflicto de intereses en el desarrollo del presente trabajo.

\section{REFERENCIAS}

1. Kobayashi Y. Neutrophil biology: an update. Excli J. 2015;14:220-227. doi:10.17179/excli2015-102

2. Brinkmann V, Reichar U, Goosmann C, Fauler B, Uhlemann Y, Weiss DS, et al. Neutrophil extracellular traps kill bacteria. Science. 2004;303:1532-1535. doi:10.1126/science.1092385303/5663/1532

3. Fuchs TA, Abed U, Goosmann C, Hurwitz R, Schulze I, Wahn V, et al. Novel cell death program leads to neutrophil extracellular traps. J Cell Biol. 2007;176:231241. doi:jcb.200606027 [pii]10.1083/jcb.200606027

4. Segal AW. How neutrophils kill microbes. Annu Rev Immunol. 2005;23:197-223. doi:DOI 10.1146/annurev. immunol.23.021704.115653

5. Guimaraes-Costa AB, Nascimento MT, Froment GS, Soares RP, Morgado FN, et al. Leishmania amazonensis promastigotes induce and are killed by neutrophil extracellular traps. Proc Natl Acad Sci. 2009;106:67486753. doi:10.1073/pnas.09002261060900226106

6. Kumar S, Gupta E, Kaushik S, Jyoti A. Neutrophil Extracellular Traps: Formation and Involvement in Disease Progression. Iran J Allergy Asthma Immunol. 2008; 17:208-220.

7. Knight JS, Kaplan MJ. Lupus neutrophils: 'NET' gain in understanding lupus pathogenesis. Curr Opin Rheumatol. 2012;24:441-450. doi:10.1097/ BOR.0b013e3283546703

8. Pinegin B, Vorobjeva N, Pinegin V. Neutrophil extracellular traps and their role in the development of chronic inflammation and autoimmunity. Autoimmun Rev. 2015;14:633-640. doi:10.1016/j.autrev.2015.03.002

9. Kambas K, Chrysanthopoulou A, Vassilopoulos D, Apostolidou E, Skendros P, Girod A, et al. Tissue factor expression in neutrophil extracellular traps and neutrophil derived microparticles in antineutrophil cytoplasmic antibody associated vasculitis may promote thromboinflammation and the thrombophilic state associated with the disease. Annals of the Rheumatic Diseases. 2014;73(10):1854-63. doi:10.1136/annrheumdis-2013-203430

10. Metzler KD, Fuchs TA, Nauseef WM, Reumaux D, Roesler J, Schulze I,et al. Myeloperoxidase is required for neutrophil extracellular trap formation: implications for innate immunity. Blood. 2011;117;953959. doi:10.1182/blood-2010-06-290171 
11. Cools-Lartigue J, Spicer J, Najmeh S, Ferri L. Neutrophil extracellular traps in cancer progression. Cell Mol Life Sci. 2014;71:4179-4194. doi:10.1007/s00018-0141683-3.

12. Remijsen Q, Berghe TV, Wirawan E, Asselbergh B, Parthoens E, De Rycke R, et al. Neutrophil extracellular trap cell death requires both autophagy and superoxide generation. Cell Res. 2011;21:290-304. doi:10.1038/cr.2010.150cr2010150

13. Gupta AK, Hasler P, Holzgreve W, Gebhardt S, Hahn S. Induction of neutrophil extracellular DNA lattices by placental microparticles and IL- 8 and their presence in preeclampsia. Hum Immunol. 2005;66:11461154. doi:S0198-8859(05)00450-7 [pii]10.1016/j.humimm.2005.11.003

14. Lin AM, Rubin CJ, Khandpur R, Wang JY, Riblett M, Yalavarthi S, et al. Mast cells and neutrophils release IL-17 through extracellular trap formation in psoriasis. J Immunol. 2011;187:490-500. doi:10.4049/jimmunol.1100123 jimmunol.1100123

15. Pilsczek FH, Salina D, Poon KK, Fahey C, Yipp BG, Sibley $C D$, et al. A novel mechanism of rapid nuclear neutrophil extracellular trap formation in response to Staphylococcus aureus. J Immunol. 2016;185:74137425. doi:10.4049/jimmunol.1000675 jimmunol.1000675

16. Bianchi M, Hakkim A, Brinkmann V, Siler U, Seger RA, Zychlinsky A, et al. Restoration of NET formation by gene therapy in CGD controls aspergillosis. Blood. 2009;114:2619-2622. doi:10.1182/ blood-2009-05-221606 blood-2009-05-221606

17. Bruns $S$, Kniemeyer $O$, Hasenberg M, Aimanianda V, Nietzsche S, Thywißen A, et al. Production of extracellular traps against Aspergillus fumigatus in vitro and in infected lung tissue is dependent on invading neutrophils and influenced by hydrophobin RodA. PLoS Pathog. 2010;6(4):e1000873. doi:10.1371/journal.ppat.1000873

18. Behrendt JH, Ruiz A, Zahner H, Taubert A, Hermosilla C. Neutrophil extracellular trap formation as innate immune reactions against the apicomplexan parasite Eimeria bovis. Vet Immunol Immunopathol. 2010;133: 1-8. doi:10.1016/j.vetimm.2009.06.012 S0165-2427(09)00210-4

19. Saitoh T, Komano J, Saitoh Y, Misawa T, Takahama M, Kozaki T, et al. Neutrophil extracellular traps mediate a host defense response to human immunodeficiency virus-1. Cell Host Microbe. 2012;12:109116. doi:10.1016/j.chom.2012.05.015 S19313128(12)00201-6

20. Wardini AB, Guimaraes-Costa AB, Nascimento MT, Nadaes NR, Danelli MG, Mazur C, et al. Characterization of neutrophil extracellular traps in cats naturally infected with feline leukemia virus. J Gen
Virol. 2010;91:259-264. doi:10.1099/vir.0.014613-0 vir.0.014613-0

21. Wang Y, Li M, Stadler S, Correll S, Li P, Wang D, et al. Histone hypercitrullination mediates chromatin decondensation and neutrophil extracellular trap formation. J Cell Biol. 2009;184:205-213. doi:10.1083/ jcb.200806072 jcb.200806072

22. Papayannopoulos V, Metzler KD, Hakkim A, Zychlinsky A. Neutrophil elastase and myeloperoxidase regulate the formation of neutrophil extracellular traps. J Cell Biol. 2010;191:677-691. doi:10.1083/ jcb.201006052

23. Metzler K, Goosmann C, Lubojemska A, Zychlinsky A, Papayannopoulos V. A myeloperoxidase-containing complex regulates neutrophil elastase release and actin dynamics during NETosis. Cell Reports. 2014;8:883-896. doi:10.1016/j.celrep.2014.06.044

24. Coelho LP, Pato C, Friães A, Neumann A, von Köckritz-Blickwede $M$, Ramirez $M$, et al. Automatic determination of NET (neutrophil extracellular traps) coverage in fluorescent microscopy images. Bioinformatics. 2015;31: 2364-2370. doi:10.1093/bioinformatics/btv156

25. Rebernick R, Fahmy L, Glover C, Bawadekar M, Shim D, Holmes CL, et al. DNA Area and NETosis Analysis (DANA): a High-Throughput Method to Quantify Neutrophil Extracellular Traps in Fluorescent Microscope Images. Biol Proced Online. 2018;20(7). doi:10.1186/ s12575-018-0072-y

26. Ginley BG, Emmons T, Lutnick B, Urban CF, Segal BH, Sarder P. Computational detection and quantification of human and mouse neutrophil extracellular traps in flow cytometry and confocal microscopy. Sci Rep. 2017;7:17755. doi:10.1038/s41598-017-18099-y

27. Zhao W, Fogg DK, Kaplan MJ. A novel image-based quantitative method for the characterization of NETosis. J Immunol Methods. 2015;423:104-110. doi:10.1016/j.jim.2015.04.027

28. Najmeh, S, Cools-Lartigue J, Giannias B, Spicer J, Ferri LE. Simplified Human Neutrophil Extracellular Traps (NETs) Isolation and Handling. JoVE. 2015;98. doi:10.3791/52687

29. Gavillet M. Martinod K, Renella R, Harris C, Shapiro NI, Wagner DD, et al. Flow cytometric assay for direct quantification of neutrophil extracellular traps in blood samples. American Journal of Hematology. 2015;90(12):1155-1158. doi:10.1002/ajh.24185

30. Vong L, Sherman PM, Glogauer M. Quantification and visualization of neutrophil extracellular traps (NETs) from murine bone marrow-derived neutrophils. Methods Mol Biol. 2013;1031:41-50. doi:10.1007/9781-62703-481-4_5

31. Quinn MT, Deleo F Bokoch GM. in Methods in molecular biology. Totowa: Humana Press; 2007. 
32. Hu Y. Isolation of human and mouse neutrophils ex vivo and in vitro. Methods in Molecular Biology. 2012;844:101-113. doi:10.1007/978-1-61779-527-5_7

33. Lundqvist $\mathbf{H}$, Follin $\mathbf{P}$, Khalfan L, Dahlgren C. Phorbol myristate acetate-induced NADPH oxidase activity in human neutrophils: only half the story has been told. J Leukoc Biol. 1996;59:270-279.

34. Karlsson A, Nixon JB, McPhail LC. Phorbol myristate acetate induces neutrophil NADPH-oxidase activity by two separate signal transduction pathways: dependent or independent of phosphatidylinositol 3-kinase. J Leukoc Biol. 2000;67:396-404.

35. Kirchner T, Möller S, Klinger M, Solbach W, Laskay T, Behnen M. The Impact of Various Reactive Oxygen Species on the Formation of Neutrophil Extracellular Traps. Mediat Inflamm. 2012. doi:Artn 849136 $10.1155 / 2012 / 849136$

36. Bjornsdottir H, Welin A, Michaëlsson E, Osla V, Berg S, Christenson K, et al. Neutrophil NET formation is regulated from the inside by myeloperoxidaseprocessed reactive oxygen species. Free Radical Bio Med. 2015;89:1024-1035. doi:10.1016/j.freeradbiomed.2015.10.398

37. Keshari RS, Verma A, Barthwal MK, Dikshit M. Reactive oxygen species-induced activation of ERK and p38 MAPK mediates PMA-inducedNETs release from human neutrophils. Journal of Cellular Biochemistry. 2013;114: 532-540. doi:10.1002/jcb.24391

38. Parker H, Dragunow M, Hampton M, Kettle AJ, Winterbourn CC. Requirements for NADPH oxidase and myeloperoxidase in neutrophil extracellular trap formation differ depending on the stimulus. J Leukoc Biol. 2012; 92:841-849. doi:10.1189/jlb.1211601

39. Arai Y, Nishinaka Y, Arai T, Morita M, Mizugishi K, Adachi S, et al. Uric acid induces NADPH oxidaseindependent neutrophil extracellular trap formation. Biochem Biophys Res Commun. 2014;443:556-561. doi:10.1016/j.bbrc.2013.12.007

40. Gupta AK, Giaglis S, Hasler P, Hahn S. Efficient neutrophil extracellular trap induction requires mobilization of both intracellular and extracellular calcium pools and is modulated by cyclosporine A. PloS One. 2014:9(5). e97088. doi:10.1371/journal.pone.0097088
41. Köckritz-Blickwede M, Chow O, Ghochani M, Chow O, Ghochani M, Nizet V. Visualization and functional evaluation of phagocyte extracellular traps. Methods in Microbiology. 2010;37:139-160.

42. Rodriguez-Espinosa O, Rojas-Espinosa O, MorenoAltamirano MM, Lopez-Villegas EO, Sanchez-Garcia FJ. Metabolic requirements for neutrophil extracellular traps formation. Immunology. 2015;145:213-224. doi:10.1111/imm.12437

43. Urban CF, Ermert D, Schmid M, Abu-Abed U, Goosmann C, Nacken W, et al. Neutrophil extracellular traps contain calprotectin, a cytosolic protein complex involved in host defense against Candida albicans. PLoS Pathog. 2009; 5(10). e1000639. doi:10.1371/journal.ppat.1000639

44. Krautgartner WD, Klappacher M, Hannig M, Obermayer A, Hartl D, Marcos V, et al. Fibrin mimics neutrophil extracellular traps in SEM. Ultrastructural Pathology. 2010;34:226-231. doi:10.3109/01913121003725721

45. Lebaron P, Catala P, Parthuisot N. Effectiveness of SYTOX Green stain for bacterial viability assessment. Applied and Environmental Microbiology. 1998;64:26972700.

46. Marin-Esteban, V, et al. Afa/Dr diffusely adhering Escherichia coli strain C1845 induces neutrophil extracellular traps that kill bacteria and damage human enterocyte-like cells. Infection and Immunity. 2012;80(5):1891-9. doi:10.1128/IAI.00050-12

47. Barrientos L, Marin-Esteba V, de Chaisemartin L, Sandre C, Bianchini E, Nicolas V, et al. An improved strategy to recover large fragments of functional human neutrophil extracellular traps. Frontiers in Immunology. 2013;4:66. doi:10.3389/fimmu.2013.00166

48. Brinkmann V, Goosmann C, Kuhn LI, Zychlinsky A. Automatic quantification of in vitro NET formation. Frontiers in Immunology; 2012;3:413. doi:10.3389/ fimmu.2012.00413

49. Gonzalez AS, Bardoel BW, Harbort CJ, Zychlinsky A. Induction and quantification of neutrophil extracellular traps. Methods in Molecular Biology. 2014;1124:307-318. doi:10.1007/978-1-62703-8454_20 